\title{
COMPOSIÇÃO CORPORAL E HÁBITOS ALIMENTARES DA POPULAÇÃO ADULTA DA REGIÃO AUTÓNOMA DA MADEIRA
}

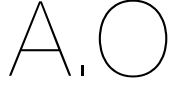

ARTIGO ORIGINAL

1 Unidade de Nutrição e Dietética do Serviço de Endocrinologia do Serviço de Saúde da Região Autónoma da Madeira, E.P.E.

Rua das Hortas, n. ${ }^{\circ} 67$, 9054-526, Funchal,

Portugal

2 Unidade de Investigação Dra. Maria Isabel Mendonça do Hospital Dr. Nélio Mendonça do Serviço de Saúde da Região Autónoma da Madeira, E.P.E., Av. Luís de Camões, n. ${ }^{\circ} 57$ 9004-514, Funchal, Portugal

Endereço para correspondência: Liliane Costa Centro de Saúde da Ribeira

Brava

Portugal

liliane.lilocosta@gmail.com

Histórico do artigo:

Recebido a 22 de julho de 201 Aceite a 20 de dezembro de 2019

\author{
BODY COMPOSITION AND FOOD HABITS OF THE ADULT \\ POPULATION OF THE AUTONOMOUS REGION OF MADEIRA
}

Liliane Costa1"; Eva Henriques²; Mariana Rodrigues²; Teresa Esmeraldo'

\section{RESUMO}

INTRODUÇÃo: Os hábitos alimentares inadequados são responsáveis pelo aumento de doenças crónicas em todo o Mundo. O diagnóstico do estado nutricional e a recolha de informação do consumo alimentar são fundamentais na definição de estratégias de promoção de uma alimentação mais saudável.

OBJETIVOS: Caracterizar o estado nutricional da população adulta (18-65 anos) da Região Autónoma da Madeira; e comparar o consumo alimentar com as recomendações da Roda dos Alimentos Portuguesa.

METODOLOGIA: Estudo transversal com 933 adultos, realizado entre 2012-2015. Dados sobre a ingestão alimentar obtidos através do questionário das 24 horas anteriores e recolhidos dados de Peso, Estatura, Perímetro da Cintura, Massa Gorda e Massa Isenta de Gordura. A análise estatística descritiva incluiu frequências, mediana, percentis 25 e 75, média e desvio-padrão. O teste do Qui-quadrado foi usado para comparar variáveis categóricas. Os testes t de Student e ANOVA foram realizados para comparar diferenças entre as médias, e os testes de Mann-Whitney e Kruskal-Wallis quando os pressupostos da normalidade não se verificaram. Foi usado um nível de significância de 5\%.

RESULTADOS: O valor médio de Índice de Massa Corporal para o total da população adulta da Região Autónoma da Madeira foi de $26,6 \mathrm{Kg} / \mathrm{m}^{2}( \pm 5,4)$, sem diferença significativa entre sexos ( $\left.p>0,05\right)$. O valor médio do Perímetro da Cintura foi de $89,2 \mathrm{~cm}( \pm 13,8)$ para as mulheres e de 93,6 cm $( \pm 13,0)$ para os homens $(p<0,05)$. O grupo etário acima dos 49 anos foi o que apresentou valores mais elevados de Peso, Índice de Massa Corporal, Massa Gorda e Perímetro da Cintura, enquanto os mais novos apresentaram os valores médios mais baixos $(p<0,05)$. Comparativamente com as recomendações, a população adulta da Região Autónoma da Madeira consumiu, proporcionalmente, mais 7\% de alimentos do grupo "Carne, pescado e ovos", e menos "Hortícolas" (-12\%), "Cereais" (-7\%) e "Fruta" (-5\%)

CONCLUSÕES: A população adulta da Região Autónoma da Madeira apresentou um consumo inadequado para quase todos os grupos de alimentos e mais de metade tinha excesso de peso.

\section{PALAVRAS-CHAVE}

Hábitos alimentares, Parâmetros antropométricos, População adulta

ABSTRACT

INTRODUCTION: Inadequate eating habits are responsible for the increase in chronic diseases worldwide. The diagnosis of nutritional status and the collection of food consumption information from the population are fundamental in the definition of a strategy to promote a healthier diet.

OBJECTIVES: To characterize the nutritional status of the adult population (18-65 years) of the Autonomous Region of Madeira; and to evaluate the adequacy of food group consumption in comparison to the Portuguese Food Wheel guide recommendation. METHODOLOGY: Cross-sectional study with 933 adults, carried out between 2012-2015. Food intake information collected by the 24hour dietary recall method and collected data on Weight, Height, Waist Circumference, Fat Mass and Fat Free Mass. The descriptive statistical analysis included frequencies, median, percentiles 25 and 75, mean and standard deviation. The chi-square test was used to compare categorical variables. Student's t-tests and ANOVA were performed to compare differences between means, and the Mann-Whitney and Kruskal-Wallis tests when normality assumptions were not verified. A significance level of $5 \%$ was used. RESULTS: The mean Body Mass Index for the total adult population of the Autonomous Region of Madeira was $26.6 \mathrm{Kg} / \mathrm{m}^{2}( \pm 5.4)$, with no significant difference between sexes ( $p>0.05$ ). The mean value of Waist Circumference was $89.2 \mathrm{~cm}( \pm 13.8)$ for women and $93.6 \mathrm{~cm}( \pm 13.0)$ for men $(\mathrm{p}<0.05)$. The age group above 49 years presented the highest values of weight, Body Mass Index, Fat Mass and Waist Circumference, while the younger ones ( $<30$ years) had the lowest mean values $(p<0.05)$. In comparison with the Portuguese Food Wheel guide recommendation, the adult population of the Autonomous Region of Madeira consumed proportionally more $7 \%$ of "Meat, fish and eggs" but less "Vegetables" (-12\%), "Cereals" (-7\%) and "Fruit" (-5\%)

CONCLUSIONS: The adult population of Autonomous Region of Madeira showed an inadequate consumption for almost all food groups, and more than half were overweight.

KEYWORDS

Dietary habits, Anthropometric parameters, Adult population 


\section{INTRODUÇÃO}

A Organização Mundial da Saúde (OMS) reconhece que os hábitos alimentares inadequados, associados a um estilo de vida inativo, têm conduzido ao aumento de doenças crónicas como a obesidade, diabetes, doenças cardiovasculares e alguns tipos de cancro (1). Em Portugal, e de acordo com os dados da Balança Alimentar, um instrumento analítico de natureza estatística que mede a disponibilidade de alimentos e a sua evolução, a população tem vindo a mostrar um desequilíbrio face às recomendações do guia alimentar oficial, a Roda dos Alimentos (2). Hábitos de consumo alimentar inadequados são já responsáveis por $86 \%$ da carga de doença no Sistema Nacional de Saúde e constituem um dos principais fatores de risco para o total de anos de vida saudáveis perdidos (3). Na Região Autónoma da Madeira (RAM), o único estudo dos hábitos alimentares da população adulta foi realizado em 1998-1999 (4, 5). Desde então, foram muitas as transformações sociais e demográficas que marcaram a Região. Em uma década (2001-2011), a RAM foi das que mais cresceu em população e é uma das regiões do país com menor índice de envelhecimento (6). O diagnóstico do estado nutricional e a recolha de informação do consumo alimentar da população adulta da RAM será, por isso, fundamental na definição de uma estratégia regional de promoção de uma alimentação mais saudável e segura.

\section{OBJETIVOS}

Este trabalho tem como objetivos principais: i) caracterizar o perfil antropométrico e composição corporal da população adulta (18-65 anos) da RAM, por sexo e grupo etário; ii) e avaliar a adequação da ingestão dos grupos alimentares relativamente às recomendações da Roda dos Alimentos Portuguesa.

\section{METODOLOGIA}

\section{População e Amostra}

Os dados do presente estudo provêm do Estudo dos Hábitos Alimentares da População Adulta da RAM (EHA-RAM), aprovado pela Comissão de Ética do Serviço de Saúde da RAM (SESARAM, E.P.E.), com o parecer N. ${ }^{\circ}$ 03/2012, e coordenado pela Unidade de Nutrição e Dietética, Serviço de Endocrinologia. A seleção dos participantes foi efetuada através da lista de utentes (18-65 anos) inscritos nos centros de saúde da RAM à data de 18 junho de 2012, depois de confirmada a sua concordância pelos dados dos Censos 2011. Foi elaborada inicialmente uma lista de 1570 utentes, a partir do cálculo de uma amostra aleatória (5\% de erro, 95\% de nível de confiança) para ser representativa por grupo etário (<30; 30-49; >49 anos) e por município, com acréscimo de $10 \%$ em cada lista. Ao longo do processo foram solicitados reforços de listas adicionais, perfazendo um total de 1800 utentes convocados. A todos os selecionados, foi enviada uma carta para explicação do estudo, com indicação da data, local da entrevista (o centro de saúde da área de residência) e os contactos para esclarecimentos ou alteração de datas e/ou local. Na véspera da entrevista, os utentes foram contactados telefonicamente para confirmarem a presença ou alterar data e/ou local, conforme conveniência. A taxa de participação foi de 53,8\%, com 969 adultos entrevistados entre novembro de 2012 e março de 2015. Todos os participantes entregaram o consentimento informado, devidamente assinado. Do total de inquéritos realizados foram excluídos 36, devido a respostas inválidas a questões fundamentais. A amostra final do presente estudo foi assim de 933 adultos que, apesar de não ser representativa por concelho (menos 1\%, 2\% e 9\% de participantes em 3 concelhos) nem grupo etário (diferença negativa de 6\% no grupo etário 30-49 anos), foi considerada representativa do total da população adulta residente na RAM. O questionário foi aplicado por profissionais de saúde da área da nutrição (dietista ou nutricionista) e a recolha de informação da ingestão alimentar foi efetuada pelo método retrospetivo de consumo das $24 \mathrm{~h}$ anteriores à data da realização da entrevista. A avaliação da composição corporal foi realizada pela medição de parâmetros antropométricos (Peso, Estatura, perímetro da cintura $(\mathrm{PC})$ ) e análise de bioimpedância (massa gorda (MG), massa isenta de gordura (MIG)). As medições foram realizadas através da balança de análise de impedância bioeléctrica tetrapolar Tanita (modelo TBF-300) e estadiómetro. A pesagem na balança foi realizada com o mínimo de roupa possível, pés descalços e na ausência de objetos metálicos, objetos nos bolsos e adereços. Aos participantes foi solicitado o esvaziamento da bexiga, no início da entrevista e aproximadamente 30 minutos antes da medição. Os registos foram efetuados no questionário em papel ou, em alternativa, eletronicamente através do questionário no formato ACCESS 2007, para o qual foi realizada formação prévia.

\section{Variáveis}

No presente estudo, as variáveis sociodemográficas usadas para caracterizar a população foram: sexo, grupo etário, escolaridade, empregabilidade, estado civil e zona de residência. A empregabilidade foi categorizada em: desempregado; ativo (trabalhador por conta de outrem ou por conta própria, e empregado doméstico); outros (inclui domésticos, estudante, reformados e outros). O estado civil foi categorizado em casado/união de facto ou não casado (divorciado, solteiro ou viúvo). Os municípios de residência foram agrupados em 4 zonas: Funchal; Zona Oeste (Câmara de Lobos, Ribeira Brava, Ponta do Sol e Calheta); Zona Norte (Porto Moniz, São Vicente e Santana); e Zona Leste (Machico, Santa Cruz e Porto Santo). Parâmetros como o peso, estatura e PC foram objetivamente medidos, de acordo com procedimentos padronizados que os profissionais da área da nutrição aplicam na sua atividade clínica diária e que constam no Manual de Procedimentos da Consulta de Nutrição do SESARAM, E.P.E. (7). O Índice de Massa Corporal (IMC) foi calculado pela fórmula peso/ estatura² . Os itens alimentares obtidos no questionário às $24 \mathrm{~h}$, incluindo bebidas, alimentos e receitas foram organizados posteriormente em grupos de alimentos.

\section{Análise Estatística}

A análise estatística foi efetuada através do programa IBM SPSS versão 25.0 para Windows e do Microsoft Excel 2010, com recurso às tabelas dinâmicas. A análise estatística descritiva consistiu no cálculo de mediana (Percentil 25 - Percentil 75), média e desvio-padrão (dp) no caso das variáveis numéricas, e no cálculo de frequências absolutas (n) e relativas (\%) no caso das variáveis categóricas. O teste de Kolmogorov-Smirnov foi usado para determinar se as variáveis estavam normalmente distribuídas na população e, nos casos em que não se verificou a normalidade, usaram-se os testes não paramétricos. Utilizou-se o teste do Qui-quadrado para comparar variáveis categóricas. Os testes $t$ de Student e ANOVA foram realizados para comparar as diferenças entre as médias e os testes de Mann-Whitney e Kruskal-Wallis quando os pressupostos da normalidade não se verificavam. Em caso de diferenças significativas entre grupos, foram realizados testes post hoc (Tukey e Tamhane). Em todas as análises, rejeitou-se a hipótese nula, quando o nível de significância crítico para a sua rejeição ( $p$ ) foi inferior a 0,05.

\section{RESULTADOS}

As características sociodemográficas da população em estudo estão descritas na Tabela 1. Do total da amostra, $61,2 \%$ eram mulheres e o grupo etário mais prevalente foi o dos 30-49 anos de idade (43,3\%). A 
Tabela 1

Características da população adulta residente na RAM

\begin{tabular}{lc}
\multicolumn{1}{l}{ TOTAL (N=933) } \\
Sexo & \\
\hline Masculino & $38,8 \%(362)$ \\
\hline Feminino & $61,2 \%(571)$ \\
\hline Grupo etário & \\
\hline$<30$ anos & $27,4 \%(256)$ \\
\hline $30-49$ anos & $43,3 \%(404)$ \\
\hline$>49$ anos & $29,3 \%(273)$ \\
\hline Escolaridade & \\
\hline$<4$ anos & $3,1 \%(29)$ \\
\hline $4-8$ anos & $36,7 \%(342)$ \\
\hline $9-12$ anos & $44,2 \%(412)$ \\
\hline$>12$ anos & $16,1 \%(150)$ \\
\hline Empregabilidade & \\
\hline Desempregado & $22,0 \%(205)$ \\
\hline Ativo & $55,5 \%(518)$ \\
\hline Outros & $22,5 \%(210)$ \\
\hline Estado civil & \\
\hline Casado/união de facto & $27,9 \%(260)$ \\
\hline Não casado & $51,9 \%(484)$ \\
\hline Residência & $48,1 \%(449)$ \\
\hline Funchal & \\
\hline Zona Oeste & \\
\hline Zona Norte & \\
\hline Zona Leste & \\
\hline & \\
\hline & \\
\hline & \\
\hline
\end{tabular}

maioria da população em estudo cumpriu 9 ou mais anos de escolaridade $(60,3 \%)$ e encontrava-se em situação de emprego ativo (55,5\%).

Os parâmetros antropométricos e os valores da composição corporal por bioimpedância são apresentados na Tabela 2. O valor médio de IMC para o total da população adulta da RAM em estudo foi de $26,6 \mathrm{Kg} / \mathrm{m}^{2}( \pm 5,4)$, sem diferença significativa entre sexos ( $\left.p>0,05\right)$. Metade da população apresentou um IMC superior a $26,1 \mathrm{~kg} / \mathrm{m}^{2}$, conforme indicado pelo valor da mediana. O valor médio do PC foi de $89,2 \mathrm{~cm}( \pm 13,8)$ para as mulheres e de $93,6 \mathrm{~cm}( \pm 13,0)$ para os homens $(p<0,0001)$. Na distribuição das variáveis antropométricas pelos grupos etários, há diferenças significativas para os valores de Peso, Estatura, IMC, MG e PC (Tabela 2). O grupo mais velho (>49 anos) foi o que apresentou valores mais elevados de Peso, IMC, MG e PC, enquanto os mais novos ( $<30$ anos) apresentaram os valores médios mais baixos $(p<0,0001)$.

A Tabela 3 descreve a distribuição do consumo habitual dos principais grupos alimentares e compara a distribuição percentual do consumo alimentar com as recomendações nacionais da Roda dos Alimentos. O consumo de "Fruta" e de "Hortícolas" pela população adulta da RAM foi, em média, de $153 \mathrm{~g} /$ dia e $43 \mathrm{~g} /$ dia, respetivamente. Os participantes reportaram um consumo médio de $78 \mathrm{~g} /$ dia de alimentos do grupo da "Carne, pescado e ovos". Dos alimentos que não fazem parte da Roda dos Alimentos Portuguesa, salienta-se que mais de metade da população adulta ingeriu quantidades superiores a $200 \mathrm{~g}$ de bebidas alcoólicas e refrigerantes/néctares. Considerando a quantidade total de alimentos e bebidas consumidos pelos indivíduos (exceto água), a percentagem de consumo dos grupos alimentares foi de $7 \%$

\section{Tabela 2}

Parâmetros de composição corporal do total da população, por sexo e grupo etário

\begin{tabular}{|c|c|c|c|c|c|c|c|c|}
\hline & \multirow{2}{*}{ TOTAL } & \multicolumn{2}{|c|}{ SEXO } & \multirow[b]{2}{*}{ p-VALUE } & \multicolumn{3}{|c|}{ GRUPO ETÁRIO } & \multirow[b]{2}{*}{ p-VALUE } \\
\hline & & MASCULINO & FEMININO & & $<30$ ANOS & 30-49 ANOS & $>49$ ANOS & \\
\hline Peso (n) & 928 & 360 & 568 & & 255 & 401 & 272 & \\
\hline Média $( \pm d p)(k g)$ & $71,5( \pm 15,0)$ & $78,3( \pm 14,5)$ & $67,2( \pm 13,7)$ & $<0,0001^{*}$ & $66,4( \pm 14,2)$ & $72,6( \pm 14,7)$ & $74,7( \pm 15,1)$ & $<0,0001^{\star}$ \\
\hline Mediana (P25-P75) (kg) & $70,0(60,2-80,5)$ & $77,3(68,7-86,0)$ & $64,9(57,5-74,5)$ & $<0,0001^{\star}$ & $63,8(55,9-73,9)$ & $70,6(61,5-81,4)$ & $73,5(63,9-82,4)$ & $<0,0001^{*}$ \\
\hline Estatura (n) & 929 & 360 & 569 & & 255 & 401 & 273 & \\
\hline Média ( $\pm \mathrm{dp})(\mathrm{cm})$ & $164,0( \pm 9,0)$ & $171,5( \pm 7,2)$ & $159,2( \pm 6,5)$ & $<0,0001^{*}$ & $166,1( \pm 9,1)$ & $164,7( \pm 8,5)$ & $160,9( \pm 9,0)$ & $<0,0001^{*}$ \\
\hline Mediana (P25-P75) (cm) & $163,1(157,6-170,0)$ & $171,0(166,6-176,0)$ & $159,0(155,0-164,0)$ & $<0,0001^{*}$ & $164,5(160,0-172,0)$ & $164,0(158,0-170,0)$ & $160,0(154,0-167,9)$ & $<0,0001^{\star}$ \\
\hline IMC (n) & 928 & 360 & 568 & & 255 & 401 & 272 & \\
\hline Média $( \pm d p)\left(k g / m^{2}\right)$ & $26,6( \pm 5,4)$ & $26,8( \pm 5,5)$ & $26,5( \pm 5,3)$ & 0,421 & $24,1( \pm 4,5)$ & $26,8( \pm 5,5)$ & $28,8( \pm 4,9)$ & $<0,0001^{\star}$ \\
\hline Mediana (P25-P75) $\left(\mathrm{kg} / \mathrm{m}^{2}\right)$ & $26,1(22,8-29,6)$ & $26,6(23,4-29,2)$ & $25,8(22,5-29,7)$ & 0,206 & $23,2(20,7-26,2)$ & $26,1(23,2-29,2)$ & $28,4(25,4-31,8)$ & $<0,0001^{\star}$ \\
\hline$M G(n)$ & 877 & 333 & 544 & & 241 & 384 & 252 & \\
\hline Média $( \pm d p)(\%)$ & $28,1( \pm 9,5)$ & $22,2( \pm 7,8)$ & $31,7( \pm 8,5)$ & $<0,0001^{*}$ & $23,1( \pm 9,4)$ & $28,7( \pm 9,0)$ & $31,9( \pm 8,2)$ & $<0,0001^{*}$ \\
\hline Mediana (P25-P75) (\%) & $28,0(21,6-35,1)$ & $22,0(16,6-27,0)$ & $32,5(25,9-37,8)$ & $<0,0001^{*}$ & $23,2(15,7-30,1)$ & $28,3(22,0-34,8)$ & $32,7(25,6-37,9)$ & $<0,0001^{*}$ \\
\hline MIG (n) & 749 & 298 & 451 & & 219 & 326 & 204 & \\
\hline Média $( \pm d p)(k g)$ & $51,0( \pm 10,0)$ & $60,5( \pm 8,2)$ & $44,6( \pm 4,5)$ & $<0,0001^{*}$ & $50,6( \pm 10,5)$ & $51,4( \pm 9,9)$ & $20,7( \pm 10,0)$ & 0,613 \\
\hline Mediana (P25-P75) (kg) & $47,6(42,9-58,4)$ & $60,8(55,0-65,0)$ & $43,9(41,6-46,8)$ & $<0,0001^{*}$ & $46,4(42,8-58,4)$ & $48,9(43,0-59,1)$ & $48,0(43,0-57,3)$ & 0,508 \\
\hline $\mathrm{PC}(\mathrm{n})$ & 927 & 360 & 567 & & 253 & 401 & 273 & \\
\hline Média ( $\pm \mathrm{dp})(\mathrm{cm})$ & $90,9( \pm 13,7)$ & $93,6( \pm 13,0)$ & $89,2( \pm 13,8)$ & $<0,0001^{*}$ & $83,2( \pm 12,4)$ & $91,1( \pm 12,7)$ & $97,8( \pm 12,5)$ & $<0,0001^{*}$ \\
\hline Mediana (P25-P75) (cm) & $90,0(80,0-100,0)$ & $93,0(83,8-102,0)$ & $87,5(79,0-99,0)$ & $<0,0001^{*}$ & $80,0(75,0-90,5)$ & $90,0(81,0-99,5)$ & $98,5(89,0-106,0)$ & $<0,0001^{*}$ \\
\hline $\begin{array}{l}{ }^{*} \text { Diferença estatística entre } \\
\% \text { : Percentagem } \\
\mathrm{cm} \text { : Centímetros }\end{array}$ & e entre gru & $\begin{array}{l}\text { IMC: Índice } \\
\text { Kg: Kilogra } \\
\text { m: Metros }\end{array}$ & $\begin{array}{l}\text { e de Massa Corporal } \\
\text { ama }\end{array}$ & & $\begin{array}{l}\text { MIG } \\
\text { PC: }\end{array}$ & $\begin{array}{l}\text { a: Massa Gorda } \\
\text { G: Massa Isenta de G } \\
\text { Perímetro da Cintura }\end{array}$ & ordura & \\
\hline
\end{tabular}


Tabela 3

Consumo habitual de alguns grupos de alimentos e comparação com as recomendações da Roda dos Alimentos

\begin{tabular}{|c|c|c|c|c|}
\hline GRUPOS DE ALIMENTOS & MÉDIA ( $\pm \mathrm{DP})(\mathrm{G} / \mathrm{DIA})$ & MEDIANA (P25-P75) (G/DIA) & CONSUMO $(\%)^{*}$ & $\begin{array}{l}\text { DIFERENÇA (\%) DAS } \\
\text { RECOMENDAÇÕES }\end{array}$ \\
\hline \multicolumn{5}{|l|}{ Da Roda dos Alimentos } \\
\hline Fruta & $153,1( \pm 87,3)$ & $150,0(100,0-174,0)$ & 15 & -5 \\
\hline Hortícolas & $42,5( \pm 48,5)$ & $27,0(14,0-51,0)$ & 11 & -12 \\
\hline Leguminosas & $35,3( \pm 39,1)$ & $19,6(10,0-41,0)$ & 1 & -3 \\
\hline Cereais, derivados e tubérculos & $82,2( \pm 69,3)$ & $55,0(42,0-100,0)$ & 21 & -7 \\
\hline Carne, pescado e ovos & $77,7( \pm 65,7)$ & $56,0(30,0-108,0)$ & 12 & +7 \\
\hline Produtos lácteos & $113,8( \pm 102,9)$ & $80,0(28,0-180,0)$ & 15 & -3 \\
\hline Óleos e gorduras & $9,3( \pm 13,6)$ & $6,0(4,0-10,0)$ & 2 & 0 \\
\hline \multicolumn{5}{|l|}{ Fora da Roda dos Alimentos } \\
\hline Refrigerantes e néctares & $236,9( \pm 100,5)$ & $205,0(180,0-295,0)$ & 11 & -- \\
\hline Bebidas alcoólicas & $240,0( \pm 237,6)$ & $200,0(120,0-250,0)$ & 7 & -- \\
\hline Bolachas, bolos e doces & $28,5( \pm 51,6)$ & $8,0(4,0-25,0)$ & 4 & -- \\
\hline Snacks, salgados e pizzas & $118,1( \pm 109,9)$ & $83,0(30,0-180,0)$ & 1 & -- \\
\hline
\end{tabular}

${ }^{*}$ Consumo alimentar em percentagem (\%) da quantidade total de alimentos consumidos DP: Desvio-padrão

de "Cereais, derivados e tubérculos", 15\% de "Fruta" e "Produtos lácteos", 12\% de "Carne, pescado e ovos", 11\% de "Hortícolas", 2\% de "Gorduras" e 1\% de "Leguminosas" (Tabela 3). Comparativamente com as recomendações preconizadas pela Roda dos Alimentos Portuguesa, a população adulta da RAM consumiu, entre 2013-2014, mais $7 \%$ do grupo "Carne, pescado e ovos", e menos 12\% de "Produtos hortícolas", $7 \%$ de "Cereais, derivados e tubérculos" e $5 \%$ de "Fruta". Os alimentos do grupo "Óleos e gorduras" foram consumidos na proporção recomendada (2\%). O conjunto de alimentos não incluídos no guia alimentar teve um contributo percentual de $23 \%$, dos quais se destaca o grupo dos "Refrigerantes e néctares" (11\%) e "Bebidas alcoólicas" (7\%).

\section{DISCUSSÃO DOS RESULTADOS}

A informação recolhida no EHA-RAM 2012-2015 não permite estabelecer comparações sobre o estado nutricional com o estudo anterior (1998-1999), principalmente por diferenças de amostragem. Em 1998/1999, foram inquiridos 920 adultos (18-74), dos quais 58,8\% apresentava excesso de peso e destes, 22,7\% eram obesos (4). É reconhecido que a média de IMC e prevalência de obesidade tendem a aumentar com a idade, mas há também evidência de alterações no padrão de composição corporal e na distribuição de gordura em idosos $(9,10)$. No EHA-RAM 2012-2015, mais de metade da população apresentou excesso de peso, segundo os critérios da OMS (11), para ambos os sexos, e a média de IMC foi superior para os indivíduos mais velhos. O IMC $\geq 25 \mathrm{~kg} / \mathrm{m}^{2}$ é um indicador de risco de comorbilidades associadas à obesidade, que deve ser usado em associação com outros índices, nomeadamente o PC, para determinar o risco cardiometabólico $(11,12)$. Um risco aumentado devido à gordura abdominal $(P C>80$ cm para mulheres; >94 cm para homens), constitui um dos principais componentes da síndrome metabólica, cuja condição aumenta 1,5 vezes o risco de mortalidade por todas as causas, e 2 vezes o risco de desenvolver doenças cardiovasculares e cerebrovasculares $(13,14)$. No presente estudo, as mulheres apresentaram, em média, um maior risco cardiometabólico, devido a PC aumentado $(>80 \mathrm{~cm})$, quando comparado com os homens (ponto de corte de $>94 \mathrm{~cm}$ ). Para ambos os índices, o risco surgiu aumentado para os grupos etários mais velhos, comparativamente aos adultos com $<30$ anos de idade. As mulheres com idade igual ou superior a 30 anos devem, por isso, ser consideradas um grupo de risco para complicações metabólicas. No que respeita aos resultados obtidos através da análise de bioimpedância, os resultados do nosso estudo são consistentes com a evidência presente na literatura, de que a MG aumenta com a idade e é maior em mulheres do que em homens (15-18).

No final da década de 90 , o estudo dos hábitos alimentares permitiu concluir que a população com 18 ou mais anos de idade tinha um consumo adequado de alimentos pertencentes aos grupos dos "Cereais, derivados e tubérculos" e "Carne, peixe e ovos", mas insuficiente dos alimentos dos grupos de "Hortícolas", "Fruta", "Leguminosas" e "Produtos lácteos" (5). Os resultados do EHA-RAM 2012-2015 apresentaram um consumo inadequado de todos os grupos de alimentos, comparativamente ao que são as recomendações, exceto para o grupo dos "Óleos e gorduras". O padrão de consumo foi caracterizado por um excesso de produtos alimentares do grupo da "Carne, pescado e ovos" e por um défice de "Hortícolas", "Fruta", "Cereais, derivados e tubérculos" e "Leguminosas". A aparente diferença na última década na adequação do consumo de "Cereais, derivados e tubérculos" e "Carne, peixe e ovos", pode dever-se ao facto de, no estudo anterior, estarem incluídos idosos que, tradicionalmente, consomem mais produtos em natureza.

Os resultados mais recentes do Inquérito Alimentar Nacional e de Atividade Física (IAN-AF 2015-2016), para o total da população portuguesa, revelam um consumo, comparativamente às recomendações, proporcionalmente superior de "Carne, pescado e ovos" (12\%) e menor de "Hortícolas" (-8\%), "Fruta" (-7\%) e "Leguminosas" (-2\%) (19). À semelhança do EHA-RAM 2012-2015, também o IAN-AF 2015-2016 revelou um consumo adequado apenas para gorduras e óleos. Uma das diferenças evidentes entre os dois estudos foi o consumo de laticínios, proporcionalmente superior no IAN-AF 2015-2016 (6\%), e inferior ao preconizado pela Roda dos Alimentos (-3\%), no EHA-RAM 2012-2015. O consumo de fruta e produtos hortícolas é considerado um dos principais indicadores da qualidade da alimentação e uma ingestão mínima diária de $400 \mathrm{~g}$ é a porção recomendada pela OMS, como estratégia de prevenção de doenças cardiovasculares, obesidade, diabetes tipo 2 e alguns tipos de cancro $(20,21)$. Na RAM, e segundo o nosso estudo, a ingestão de fruta, hortícolas e leguminosas, pela população adulta, perfaz cerca de $230 \mathrm{~g}$, o que corresponde a pouco mais de metade do que é recomendado. Resultados semelhantes foram obtidos no IAN-AF 2015-2016, onde a RAM se destacou por ter uma das prevalências mais elevadas de inadequação de fruta e hortícolas. As estratégias regionais de promoção de uma alimentação saudável devem, por isso, fomentar o consumo habitual destes produtos alimentares. O consumo de "Carne, pescado e ovos" parece ter aumentado nos últimos anos na RAM, passando de uma ingestão adequada (1998/1999) 
para um consumo excessivo ( $+7 \%$ do que o recomendado no EAH-RAM 2012-2015). Contudo, no panorama Nacional, os dados mais recentes (IAN-AF 2015-2016) revelam que a Madeira foi a região do país com prevalências de inadequação de carnes vermelhas e processadas mais baixas. Este resultado pode surpreender, uma vez que a carne vermelha é um ingrediente fundamental no típico prato "espetada", globalmente difundido entre a população da RAM. O reconhecimento da Agência Internacional de Investigação em Cancro de que este tipo de carnes está associada positivamente a cancro em alguns órgãos humanos, nomeadamente cólon, pâncreas, estômago e próstata, enfatiza o consumo moderado de carnes vermelhas e processadas (22). No geral, iniciativas de promoção de uma ingestão adequada de "Carne, pescado e ovos" devem ser integradas na estratégia regional de promoção de uma alimentação mais saudável e segura.

No EHA-RAM 2012-2015, uma população mais jovem aliada ao aumento da disponibilidade de alimentos do tipo fast-food e processados, pode ter potenciado o consumo destes últimos, em detrimento dos alimentos mais tradicionais. Neste estudo, a ingestão média de "Snacks, salgados e pizzas" foi superior à média consumida de "Cereais, derivados e tubérculos" e "Carne, pescado e ovos". O contributo calórico total dos grupos de alimentos não incluídos na Roda dos Alimentos foi, em média, de cerca de $800 \mathrm{Kcal} / \mathrm{dia}$, com destaque para as bebidas "Refrigerantes e néctares" e "Bebidas alcoólicas", o que pode constituir um fator importante para a prevalência de excesso de peso no total da população adulta e, em particular, no excesso de gordura abdominal no sexo feminino.

O presente estudo apresenta pontos fortes e também algumas limitações. Como ponto forte, destaca-se a representatividade da amostra a nível regional para a população adulta. Trata-se do segundo estudo de base populacional realizado na RAM, sobre hábitos alimentares e estado nutricional em adultos, e os seus resultados serão fundamentais para a definição de estratégias de saúde pública. Outro aspeto importante é que a recolha de dados foi realizada por profissionais da área da Nutrição treinados, conferindo maior precisão dos valores do que no caso de dados autoreportados. Uma das principais limitações deste estudo prende-se com a falta de representatividade da amostra por grupo etário e por município. Adicionalmente, é notória a discrepância entre os participantes no que respeita ao sexo (61\% mulheres vs. 39\% homens), pelo que é necessária cautela no tratamento e interpretação dos dados.

\section{CONCLUSÕES}

No EHA-RAM 2012-2015, mais de metade da população adulta (18-65 anos) apresentou excesso de peso. As mulheres com idade igual ou superior a 30 anos constituem um grupo de risco para complicações metabólicas, associadas à gordura abdominal. Comparativamente ao que são as recomendações, quase todos os grupos de alimentos apresentaram um consumo inadequado. A estratégia regional de promoção da alimentação saudável e segura deve, por isso, promover o aumento do consumo de "Hortícolas", "Fruta" e "Leguminosas", assim como de "Cereais e derivados, tubérculos" e "Produtos Lácteos". Ao mesmo tempo devem ser encetados esforços para reduzir a ingestão de bebidas densamente calóricas e pobres sob o ponto de vista nutricional, nomeadamente "Refrigerantes e néctares" e "Bebidas alcoólicas".

\section{AGRADECIMENTOS}

Um agradecimento especial ao Centro de Investigação Dr. ${ }^{a}$ Maria Isabel Mendonça e ao Núcleo de Gestão de Doentes e Estatística do Serviço de Saúde da Região Autónoma da Madeira, E.P.E. pela contribuição na análise estatística dos dados.

\section{REFERÊNCIAS BIBLIOGRÁFICAS}

1. World Health Organization, Regional Office for Europe. European food and nutrition action plan 2015-2020. Copenhagen: World Health Organization, Regional Office for Europe; 2015. 2. Rodrigues SSP, Franchini B, Graça P, Almeida MDV de. A New Food Guide for the Portuguese Population: Development and Technical Considerations. Journal of Nutrition Education and Behavior. 2006 May 1 [cited 2018 Mar 25];38(3):189-95. Available from: http://www.jneb.org/article/S1499-4046(06)00030-3/fulltext.

3. Portugal. Direção-Geral da Saúde., Direção de Serviços de Informação e Análise. A Saúde dos Portugueses 2016. Direção-Geral da Saúde; 2017.

4. Gabiente para a Qualidade e Investigação. Diagnóstico da situação alimentar e nutricional da Região Autónoma da Madeira. Funchal, Região Autónoma da Madeira: Secretaria Regional dos Assuntos Sociais; 2000.

5. Secretaria Regional dos Assuntos Sociais. Plano de Saúde. Direcção Regional de Planeamento e Saúde Pública, Direcção de Serviços de Planeamento; 2003.

6. Instituto Nacional de Estatística. Censos 2011 Resultados Definitivos - Região Autónoma da Madeira. Lisboa: INE, I.P.; 2012.

7. Secretaria Regional dos Assuntos Sociais. Manual de Procedimentos da Consulta de Nutrição. Funchal, Região Autónoma da Madeira: Direção Regional de Planeamento e Saúde Pública; 2007. 37 p.

8. Secretaria Regional dos Assuntos Sociais, Direção Regional de Planeamento e Saúde Pública. Manual de Procedimentos da Consulta de Nutrição. DRPSP; 2007.

9. Flegal KM, Carroll MD, Kit BK, Ogden CL. Prevalence of obesity and trends in the distribution of body mass index among US adults, 1999-2010. Jama. 2012;307(5):491497. doi: 10.1001/jama.2012.39.

10. Vlassopoulos A, Combet E, Lean ME. Changing distributions of body size and adiposity with age. International journal of obesity. 2014;38(6):857. doi: 10.1038/jo.2013.216.

11. WHO, IASO, IOFT. WHO | The Asia-Pacific Perspective: Redefining obesity and its treatment [Internet]. WHO. 2000 [cited 2017 Feb 20]. Available from: http://www. who.int/nutrition/publications/obesity/09577082_1_1/en/.

12. Organization WH. Obesity: preventing and managing the global epidemic: report of a WHO consultation on obesity, Geneva, 3-5 June 1997. Geneva: World Health Organization; 1998. 13. World Health Organization. Waist circumference and waist-hip ratio: report of a WHO expert consultation, Geneva, 8-11 December 2008. Geneva: World Health Organization; 2011. 14. Engin A. The Definition and Prevalence of Obesity and Metabolic Syndrome. Adv Exp Med Biol. 2017;960:1-17. doi: 10.1007/978-3-319-48382-5_1.

15. He X, Li Z, Tang X, Zhang L, Wang L, He Y, et al. Age-and sex-related differences in body composition in healthy subjects aged 18 to 82 years. Medicine. 2018;97(25). doi: 10.1097/MD.0000000000011152.

16. Amoo-Tella SY, Danborno BS, Akuyam S, Adebisi SS. Gender-and age-related differences in anthropometric and body composition parameters in Nigerians, Zaria, Nigeria. Journal of Experimental and Clinical Anatomy. 2017;16(2):137. doi: 10.4103/jeca.jeca_4_17.

17. Kirchengast S. Gender differences in body composition from childhood to old age: an evolutionary point of view. Journal of Life Sciences. 2010;2(1):1-10. https://doi.org /10.1080/09751270.2010.11885146.

18. Kyle UG, Genton L, Slosman DO, Pichard C. Fat-free and fat mass percentiles in 5225 healthy subjects aged 15 to 98 years. Nutrition. 2001;17(7-8):534-541. https:// doi.org/10.1016/S0899-9007(01)00555-X.

19. Lopes C, Torres D, Oliveira A, Severo M, Alarcão V, Guiomar S, et al. Inquérito Alimentar Nacional e de Atividade Física 2015-2016: Relatório dos resultados. Universidade do Porto. 2017.Available online: https://ian-af.up.pt/ [cited 2020 Jan 14]. 20. World Health Organization. Diet, Nutrition and the Prevention of Chronic Diseases: Report of a Joint WHO/FAO Expert Consultation. Geneva. 2003. WHO technical report series. [cited 2020 Jan 14]. Available from: http://apps.who.int/iris/ bitstream/10665/42665/1/WHO_TRS_916.pdf?ua=1.

21. World Health Organization. Fruit and Vegetable Promotion Initiative: A Meeting Report, 25-27 August, Geneva. [cited 2020 Jan 14] Available from: http://apps.who. int/iris/bitstream/10665/68395/1/WHO_NMH_NPH_NNP_0308.pdf.

22. International Agency for Research on Cancer. Volume 114: Consumption of red meat and processed meat. IARC Working Group. Lyon; 6-13 September, 2015. IARC Monogr Eval Carcinog Risks Hum (in press). 